\title{
Laparoscopic Treatment of Hepatic Abscess Induced by Foreign Body Impaling Through the Stomach - A Case Report
}

\author{
Chun Yi Tsai ${ }^{1}$, Pei Ching Huang ${ }^{2}$, Wen Hui Chan ${ }^{2}$ and Chun Nan Yeh*1 \\ ${ }^{1}$ Department of General Surgery, Chang Gung Memorial Hospital, Chang Gung University, Taiwan \\ ${ }^{2}$ Department of Radiology, Chang Gung Memorial Hospital, Chang Gung University, Taiwan
}

Received: 㭗 June 02, 2018; Published: 監July 20, 2018

*Corresponding author: Chun Nan Yeh, Department of Surgery, Chang Gung Memorial Hospital and Chang Gung Univerity 5, Fu-Hsing Street, Kwei-Shan, Taoyuan, Taiwan, Tel: +886-3-3281200; FAX: +886-3-3285818; Email: yehchunnan@gmail.com

Abbreviations: GI: Gastrointestinal; CT: Computed Tomography

\section{Background}

Unintended ingestion of foreign body into gastrointestinal (GI) tract is not an uncommon event, and it complicates with perforation, obstruction or intra-abdominal infections in certain scenarios. The incidence of GI tract perforation developed after foreign body ingestion is less than 1\% [1]. Hepatic pyogenic abscess resulting from foreign body migration is even rare [2]. Among the reported patients, most of them failed to recall the time of ingestion and the type of foreign body. The disease severity of hepatic abscess ranged from indolent chronic infection to life-threatening septic shock, but the treatment was similarly composed of adequate drainage and targeted antibiotics [3]. Regard the rare but specific spectrum of secondary hepatic abscess induced by foreign body ingestion, removal of the migrated foreign body is crucial in controlling the infection [4]. Owing to the non-specific symptoms of hepatic abscess and the difficulty in preoperative recognition of foreign body ingestion, secondary hepatic abscess remained a diagnostic challenge. The following case represents a hepatic abscess secondary to unaware gastric perforation by a fishbone that was treated by laparoscopic removal of the foreign body and percutaneous drainage of the abscess.

\section{Case Presentation}

A 63-year-old woman presented to our hospital due to fever, chillness, decreased appetite and vague epigastric pain for 3 days after returning from Netherland for sightseeing. Laboratory examination demonstrated leukocytosis, and sonography of the liver disclosed an irregular cystic lesion within the segment IV of the liver. Owing to progressive pain and persistent fever, she was referred for computed tomography (CT) scan under the diagnosis of hepatic abscess. The composed CT scan demonstrated an abscess measuring $4.3 \times 2.7 \mathrm{~cm}$ within the segment IV of the liver, in association with a foreign body measuring $2.8 \mathrm{~cm}$ impaling from peripyloric area of the stomach (Figures 1). There was remarkable fibrotic tissue around the foreign body and space between the two impaled organs. She could not recall any episodes of fever or sharp abdominal pain during the last few weeks. Regard to the CT images, the foreign body already migrated through the gastric mucosa that endoscopic removal is not feasible. She underwent laparoscopic exploration thereafter. Marked adhesion and fibrosis between the liver and the pyloric area of the stomach was found (Figure 2A), which compatible with the preoperative CT images. The foreign body, which was a fishbone, was removed after meticulous dissection along the fibrotic tissue (Figure 2B), and the impaled site on the stomach was repaired with interrupted sutures and omentum patch (Figure 2C). Concerning the spillage of abscess into peritoneal cavity by concomitant surgical drainage, we applied a pigtail into the abscess via percutaneous fashion (Figure 2D). The bacterial culture from the abscess yielded Klebsiella pneumonia, Streptococcus anginosus, and Actinomyces odontolyticus, which were eradicated by targeted antibiotics for 5 days. She resumed oral intake since postoperative day 5 and was discharged on postoperative day 8 after removal of the drainage for the abscess. 


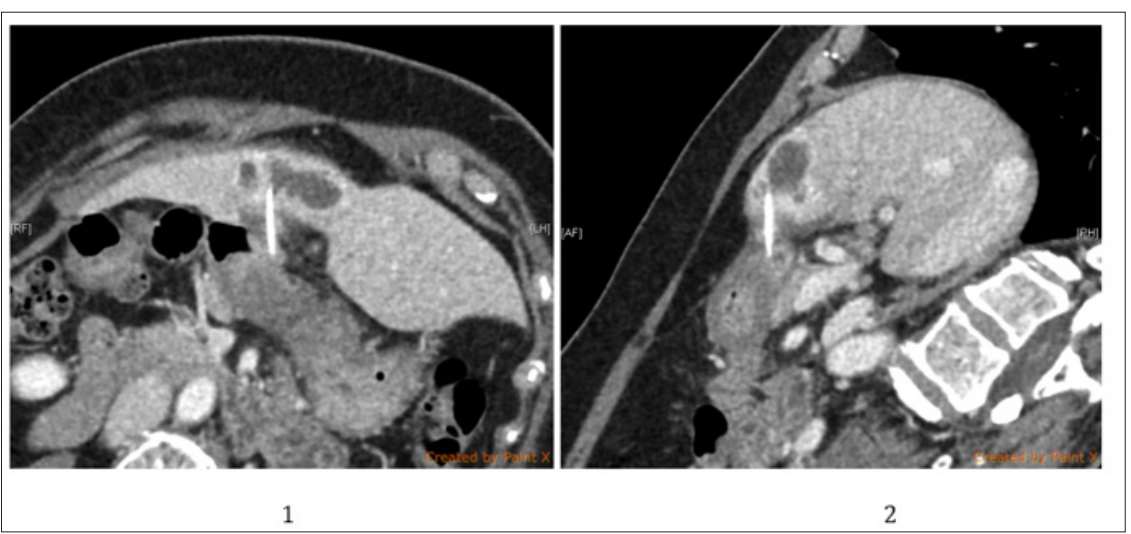

Figure 1: Abdominal CT findings.

The composed CT scan demonstrated the whole path of the foreign body between the abscess within the segment IV of the liver and the stomach in recombinant coronal and sagittal views.
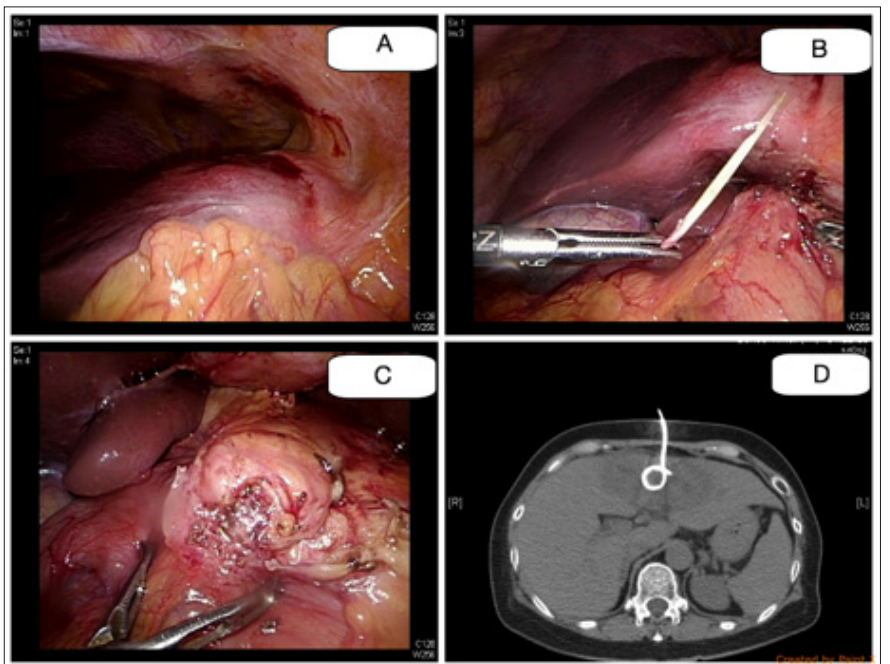

Figure 2 : The operative findings and CT findings

A. Part of the omentum and the pylorus of stomach adhered to the liver.

B. The fishbone was retrieved after meticulous dissection along the tract formed by fibrotic tissues.

C. The impaled site on the stomach demonstrated perifocal fibrotic tissue formation.

D. CT-guided drainage of the abscess by a pigtail catheter.

\section{Discussion}

Most of the ingested foreign bodies pass through GI tract spontaneously, while some of them mandate intervention [5]. Surgery is warranted for foreign body related perforation with immediate peritonitis or obstruction. Hepatic abscess secondary to perforation of stomach induced by foreign body is a specific disease entity, owing to its rarity and obscure clinical presentations [4]. The peristaltic waves pushed the sharp foreign bodies through the stomach, presumably the antrum, and resulted into gradual penetration that permit enough time for the omentum to wrapped around [6]. The foreign body impales the adjacent liver and resulted into secondary hepatic abscess. Among the reported patients [2], most of them could not recall the time of ingestion and the type of foreign body. The clinical manifestations of hepatic abscess include fever, chillness, or right upper quadrant pain, which were not specific to achieve the diagnosis. Therefore, the diagnosis of hepatic abscess resulted from foreign body which prompt adequate intervention relied on awareness of physicians and accurate image acquisition. CT scan is the preferred modality for its ability to demonstrate linear calcified structures [7].

Regard to our patient, the diagnosis was achieved after CT illustrated the straight and calcified fishbone between the liver abscess and the stomach. Stemming from the precise preoperative images, though lack of definite history, the fishbone could be removed via totally laparoscopic approach. Operative drainage contained one of the traditional treatment for hepatic abscess, which associated with high surgical morbidities [8]. The drainage method evolved with time and nowadays the majority of patients underwent image-guided percutaneous drainage with remarkable successful rates since 1990s [9,10]. Some authors also proposed drainage of hepatic abscess laparoscopically [11]; however, it remains an alternative modality in selected cases and when 
percutaneous approaches fail. In conclusion, the reported case achieved the diagnosed as foreign body induced hepatic abscess on CT images, which mandated both the foreign body and the hepatic abscess be managed in minimal invasive fashions successfully.

\section{References}

1. Goh BK, Chow PK, Quah HM, Ong HS, Eu KW, et al. (2006) Perforation of the gastrointestinal tract secondary to ingestion of foreign bodies. World J Surg 30(3): 372-377.

2. Santos SA, Alberto SC, Cruz E, Pires E, Figueira T, et al. (2007) Hepatic abscess induced by foreign body: case report and literature review. World J Gastroenterol 13(9): 1466-1470.

3. Rahimian J, Wilson T, Oram V, Holzman RS (2004) Pyogenic liver abscess: recent trends in etiology and mortality. Clin Infect Dis 39(11): 1654-1659.

4. Leggieri N, Marques Vidal P, Cerwenka H, Denys A, Dorta G, et al. (2010) Migrated foreign body liver abscess: illustrative case report, systematic review, and proposed diagnostic algorithm. Medicine (Baltimore) 89(2): 85-95.

\section{ISSN: 2574-1241}

DOI: 10.26717/BJSTR.2018.07.001454

Chun Nan Yeh. Biomed J Sci \& Tech Res

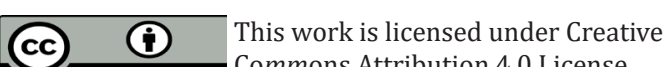

Submission Link: https://biomedres.us/submit-manuscript.php
5. Velitchkov NG, Grigorov GI, Losanoff JE, Kjossev KT (1996) Ingested foreign bodies of the gastrointestinal tract: retrospective analysis of 542 cases. World J Surg 20(8): 1001-1005.

6. Dangoisse C, Laterre PF (2014) Tracking the foreign body, a rare cause of hepatic abscess. BMC Gastroenterol 14: 167.

7. Ngan JH, Fok PJ, Lai EC, Branicki FJ, Wong J (1990) A prospective study on fish bone ingestion. Experience of 358 patients. Ann Surg 211(4): 459-462.

8. Singh S, Chaudhary P, Saxena N, Khandelwal S, Poddar DD, et al. (2013) Treatment of liver abscess: prospective randomized comparison of catheter drainage and needle aspiration. Ann Gastroenterol 26(4): 332 339.

9. Seeto RK, Rockey DC (1996) Pyogenic liver abscess. Changes in etiology, management, and outcome. Medicine (Baltimore) 75(2): 99-113.

10. Cai YL, Xiong XZ, Lu J, Cheng Y, Yang C, et al. (2015) Percutaneous needle aspiration versus catheter drainage in the management of liver abscess: a systematic review and meta-analysis. HPB (Oxford) 17(3): 195-201.

11. Wang W, Lee WJ, Wei PL, Chen TC, Huang MT (2004) Laparoscopic drainage of pyogenic liver abscesses. Surg Today 34(4): 323-325.

Assets of Publishing with us
BIOMEDICAL
RESEARCHES

